\title{
Dialekte im DaF-Unterricht? Ja, aber... \\ Konturen eines Konzepts für den Aufbau einer rezeptiven Varietätenkompetenz
}

\author{
Thomas Studer (Freiburg/ Fribourg)
}

\begin{abstract}
:
Dialekte im DaF-Unterricht? Ja, aber...

Sollen Dialekte im DaF-Unterricht berücksichtigt werden? Neuere Untersuchungen zu den Einstellungen gegenüber Varietäten des Deutschen bei DaF-Lehrenden und -Lernenden (z.B. Baßler / Spiekermann 2001) weisen darauf hin, dass bezüglich dieser Frage eine gewisse Verunsicherung besteht. Der vorliegende Beitrag reagiert auf diese Verunsicherung, indem er einen Orientierungsrahmen entwirft, der es erlaubt, den Dialekten im Unterricht positiv, aber reflektiert zu begegnen. Im Einzelnen werden folgende Fragen diskutiert: Wie lässt sich die Integration von Dialekten in den Unterricht begründen? Unter welchen Voraussetzungen und auf welchen Stufen ist es sinnvoll, Dialekte zu behandeln? Wie und in welchem Umfang könnte das erfolgen? Was genau könnte eine Dialektkompetenz beinhalten und welche sprachlichen Teilfertigkeiten sollten im Zentrum stehen? Welche (und wieviele) Dialekte sollten berücksichtigt werden? Welche Rolle spielt der Lernort? - Aus der Diskussion dieser Fragen resultiert ein Konzept für einen erweiterten Erwerb des Deutschen als Fremdsprache, zu dem an erster Stelle eine breitere Wahrnehmungstoleranz gegenüber Standardvarietäten des Deutschen gehört und der sich dann auch, im Bereich der soziokulturellen und rezeptiven Kompetenzen, auf Dialekte erstreckt.

Der Beitrag ist aus einer Deutschschweizer Perspektive geschrieben, bietet aber Kriterien an, die auf verschiedene Lernsituationen im deutschsprachigen Inland und im Ausland bezogen werden können.
\end{abstract}

\section{Zum Thema}

Dialekte im DaF-Unterricht sind (wieder) ein Thema, für wissenschaftliche Untersuchungen ebenso wie für Erfahrungsberichte (cf. etwa Baßler/Spiekermann 2002, 2001, Feuz 2001a, b, Winiger 2001, von Flüe-Fleck/Hove 1994, Studer im Druck). Doch viele Lehrerinnen und Lehrer scheinen eher verunsichert: "Und jetzt auch noch die Dialekte"?

Ein Indiz für diese Verunsicherung sind die Befunde zu den Einstellungen von DaFLehrenden in Freiburg i.Br. zu Varietäten des Deutschen aus der umsichtigen Fragebogenstudie von Baßler/Spiekermann $(2001)^{1}$ die in ganz verschiedene Richtungen weisen: Einerseits sind sich alle LehrerInnen der Variation der Zielsprache durchaus bewusst, der Dialekt wird als wichtiges Ausdrucksmittel für den Alltag angesehen und ihm werden

\footnotetext{
${ }^{1}$ Zum Sample der Untersuchung und zur realisierten Fragebogenmethode cf. Baßler/Spiekermann (2001: 5f). Befragt wurden 151 DaF-Lernende und 15 Lehrende des Sprachenkollegs für ausländische Studierende (DSHVorbereitung) und des Goethe-Instituts Inter Nationes (Prüfungsvorbereitungskurse) in Freiburg i.Br. Die Lernenden kommen aus 48 verschiedenen Ländern; sie haben eine durchschnittliche Lernerfahrung von 2 Jahren und haben im Mittel ein halbes Jahr Unterrichtszeit in Deutschland verbracht. Die Lehrenden sind mehrheitlich in Süddeutschland aufgewachsen und verfügen über langjährige Unterrichtserfahrung.
} 
auch positive Eigenschaften besonders im Bereich sozialer und zwischenmenschlicher Faktoren zugeschrieben (ibd.: 16, 10 u. 8). Andererseits ordnen die Freiburger LehrerInnen den Dialekt v. a. den "Arbeitern" und "Bauern" und dann auch den "Angestellten" zu (16) und $80 \%$ von ihnen sind der Meinung, DialektsprecherInnen würden in der Gesellschaft benachteiligt (ibd.: 14). Aufhorchen lässt dann auch, dass nur ein Viertel der Lehrenden die Fähigkeit, sich an GesprächspartnerInnen anpassen zu können, als wichtiges oder sehr wichtiges Lernziel ansieht (ibd.: 19). Insgesamt halten die befragten Freiburger LehrerInnen den Dialekt für ziemlich unbedeutend, wenn es um die Frage der zu vermittelnden Kompetenzen geht (ibd.), wobei es Unterschiede in Bezug auf die Einschätzung der Relevanz der Dialekte für die sprachlichen Teilfertigkeiten gibt (ibd.; cf. Näheres dazu weiter unten unter 3.4).

Nun sind Einstellungen als relativ feste, nicht ohne weiteres und nur in Grenzen veränderbare mentale Konstrukte, die das Handeln - und das heißt hier auch: das unterrichtliche Handeln prädisponieren, ernst zu nehmen, und zwar auch dann, wenn sie mit wissenschaftlichen Einsichten nicht im Einklang stehen. ${ }^{2}$ So spiegelt etwa die "Benachteiligungshypothese", die vier von fünf LehrerInnen vertreten, nicht die Erkenntnisse neuerer soziolinguistischer Forschung, sondern erinnert deutlich an die frühe Sprachbarrierendiskussion in den 70er Jahren, wobei z. B. schon Bausinger (1973) darauf hingewiesen hat, dass das StandardDialekt-Kontinuum, wie es in den südlichen Regionen Deutschlands zu beobachten ist, den Dialekt aus seiner einseitigen soziolektalen Zuweisung herauslöst. ${ }^{3}$

Im Folgenden sollen die von Baßler/Spiekermann (2001) beigebrachten Befunde zum Anlass genommen werden, über konzeptuelle Fragen zum Einbezug der Dialekte in den DaFUnterricht nachzudenken. Die Grundthese dieses Beitrags ist, dass die ambivalente Einschätzung des Dialekts seitens der LehrerInnen auch mit einer gewissen Verunsicherung zu tun hat, die ihrerseits auf das Fehlen einschlägiger Konzepte zur Integration der Dialekte in das Lehren und Lernen von Fremdsprachen allgemein und in den DaF-Unterricht besonders verweist.

Ziel dieses Beitrags ist es, einen ersten Orientierungsrahmen für die Berücksichtigung von Dialekten im DaF-Unterricht zu entwerfen. Dazu wird im ersten Abschnitt unter Punkt 2 kurz der Argumentationszusammenhang, i. e. die Deutschschweizer Sprachsituation, skizziert. Die darauf Bezug nehmenden, aber nicht darauf beschränkten konzeptuellen Überlegungen sind dann Gegenstand von Punkt 3. Angeordnet sind die Überlegungen nach den folgenden sieben Leitfragen:

1. Welche Argumente lassen sich für die Behandlung von Dialekten anführen?

2. Welche Rahmenbedingungen sollten für die Behandlung von Dialekten beachtet werden?

3. Auf welchen Stufen ist die Thematisierung von Dialekten sinnvoll?

4. Welche sprachlichen Teilfertigkeiten sollten im Zentrum stehen?

5. Welche Dialekte könnten Unterrichtsgegenstand sein?

6. Welche didaktischen Prinzipien bieten sich für Behandlung von Dialekten im Unterricht an?

\footnotetext{
${ }^{2}$ Das gilt ganz generell, cf. etwa Hoberg (2000) im Kontext von Überlegungen zur ablehnenden Haltung vieler Deutschen gegenüber neueren Anglizismen; zu Spracheinstellungen allgemein siehe die einschlägigen Hinweise bei Baßler/Spiekermann (2001.), zudem z. B. auch Baker (1992); zum Problem der Veränderbarkeit von Einstellungen speziell Klauer (1988).

${ }^{3}$ Der Hinweis auf Bausinger ist dem kurzen Überblick über die Diskussion um den Dialekt als Sprachbarriere bei Schmidlin (2000), Kap. 1.2.2.1, entnommen.
} 
7. In welchem Umfang sollen Dialekte behandelt werden und was für "Gefässe" eignen sich dafür?

Im letzten Abschnitt werden die Überlegungen dann noch zusammengefasst und bilanziert (Punkt 4).

\section{Die Deutschschweizer Sprachsituation als Argumentationsrahmen}

Darstellungen der Sprachsituation in der Deutschschweiz gibt es viele. Unter den aktuellen sind Haas (2001, 2000), Werlen (1998), Siebenhaar/Wyler (1997); eine leicht lesbare, für DaF-Lehrende im Ausland geschriebene Einführung findet sich auch auf der CD-ROM Grüezi mitenand, die für die IDT 2001 in Luzern produziert wurde. Diese Darstellungen werden aber hier nur insoweit berücksichtigt, als sie zum Verständnis der konzeptuellen Überlegungen unter Punkt 3 beitragen. In dieser Hinsicht wichtig sind v. a. drei Punkte:

1 In der Deutschschweiz trifft man auf eine "use oriented" Diglossie, also auf ein gebrauchsorientiertes Nebeneinander von Hochdeutsch und Dialekten, bei dem die Entscheidung für eine von den beiden Sprachformen im Wesentlichen davon abhängt, ob mündlich oder schriftlich kommuniziert wird (deshalb "mediale" oder "modale Diglossie"). In der mündlichen Kommunikation dominiert der Dialekt, und zwar unabhängig von sozialen Schichten und Berufsgruppen, d.h. nicht nur "Büezer" (ArbeiterInnen) und Bauern sprechen Dialekt, sondern auch AkademikerInnen, und zwar oft auch in formelleren Kontexten. In der schriftlichen Kommunikation hingegen dominiert das Hochdeutsche - ebenfalls weitgehend unabhängig von sozialen Schichten und Berufsgruppen. ${ }^{4}$ - Im Gegensatz dazu kann die Sprachsituation Deutschlands eher als "user oriented" Diglossie bezeichnet werden. Das wäre dann eine Diglossie, bei der bestimmte, u. a. areal unterschiedlich ausgeprägte Zusammenhänge zwischen Dialektgebrauch und sozialer Stratifizierung möglich sind.

2 In der Deutschschweiz gibt es kein Kontinuum zwischen Hochdeutsch und Dialekt. Die beiden Varietäten sind immer deutlich voneinander getrennt, Übergangsformen gibt es nicht und der Wechsel vom Dialekt in die Hochsprache (oder umgekehrt) erfolgt immer sehr bewusst. DeutschschweizerInnen kommunizieren in ihren Dialekten miteinander, auch über Dialektgrenzen hinweg. Der Dialekt ist eine Art "Standard nach innen", wohingegen der nationalen Standardvarietät des Deutschen in der Schweiz, also dem Schweizerhochdeutschen, in der gesprochenen Modalität allenfalls noch die Rolle eines "Standards nach aussen" bleibt (zu diesem Begriffspaar cf. Muhr 2000; zum gesprochenen Schweizerhochdeutsch cf. jetzt besonders Hove 2002). In deutlichem Gegensatz dazu gibt es in Deutschland verschiedene Varietäten des Deutschen, die zwischen Dialekt und Standardsprache stehen, und die als "Umgangssprachen" genutzt werden (bei Baßler/Spiekermann 2001: 2f. "Regionalsprachen" und "regionale Standards", die sich durch verschiedene Ausprägungen von "Dialektalität" unterscheiden und die im Vergleich zu den Dialekten eine grössere kommunikative Reichweite aufweisen).

3 Vor diesem Hintergrund ist es wenig überraschend, dass die Einstellungen zum Dialekt und zum Hochdeutschen in der Deutschschweiz ganz anders (und vermutlich auch

\footnotetext{
${ }^{4}$ Genau besehen ist die Verteilung von Hochdeutsch und Dialekt allerdings nicht streng komplementär. Zunehmende Verschiebungen sind heute v. a. auch bei neuen "Textsorten" wie E-Mail und SMS zu beobachten, die durchaus auch - wegen ihrem mündlich-kolloquialen Stil - im Dialekt geschrieben werden können. Umgekehrt gibt es im Bereich der gesprochenen Sprache deutliche Anzeichen für ein weiteres Schwinden ursprünglicher Hochdeutschdomänen; ein Beispiel dafür sind politische Debatten im Fernsehen. Diese Tendenzen werden oft mit dem strapazierten Begriff der "Mundartwelle" belegt - und v. a. auch von französischsprachigen SchweizerInnen immer wieder kritisiert (cf. z. B. Pilet 2001).
} 
einheitlicher) verteilt sind als in Deutschland. Die Hochwertvarietät in der Deutschschweiz ist der Dialekt, und die affektiven Einstellungen zum gesprochenen Hochdeutschen sind in der Regel alles andere als positiv ausgeprägt: DeutschschweizerInnen sprechen nicht gern Hochdeutsch, und wenn sie es trotzdem tun, was etwa noch im Kontakt mit Fremdsprachigen vorkommt, dann fühlen sie sich, so sagt man, nicht besonders wohl dabei. ${ }^{5}$

Mit Blick auf die hier nur grob skizzierte Deutschschweizer Sprachsituation mag es erstaunen, dass es bisher kaum allgemeine DaF-Lehrmittel gibt, die an diesen besonderen Erwerbskontext angepasst sind (eine Ausnahme ist das 2000 erschienene Lehrmittel "Deutsch in der Schweiz" von Ernst Maurer $)^{6}$ und dass bis jetzt von Schweizer Seite noch keine detaillierteren Konzepte für die Integration des Dialekts in den DaF-Unterricht vorgeschlagen wurden. Hier einen ersten Schritt zu tun, ist das Ziel des folgenden Kapitels.

\section{$3 \quad$ Facetten eines Konzepts für einen erweiterten Erwerb des Deutschen als Fremdsprache, der sich im Bereich der rezeptiven Kompetenzen auch auf Dialekte erstreckt}

\subsection{Warum Dialekte?}

Für die Beschäftigung mit Dialekten im DaF-Unterricht lassen sich zwei ganz verschiedene Argumente anführen, zum einen Überlegungen im Umkreis von Mehrsprachigkeitskonzeptionen und interkulturellen Lernzielen und zum andern Resultate aus Befragungen von Deutschlernenden. Verschieden zu beurteilen wären dabei nicht nur die Inland- und die Auslandsituation, sondern auch verschiedene Sprachlernsituationen innerhalb des deutschsprachigen Raumes, was hier allerdings nur in Ansätzen erfolgen kann.

\subsubsection{Mehrsprachigkeitskonzeptionen und interkulturelle Lernziele}

Allgemein begründen lässt sich die Thematisierung von Dialekten im DaF-Unterricht durch ein Mehrsprachigkeitskonzept, wie es beispielsweise im Gemeinsamen europäischen Referenzrahmen für Sprachen vertreten wird (deutsche Übersetzung des Framework, Ausgabe 2001, Kap. 1.3). Dort wird die Tatsache betont, dass sich die Spracherfahrungen eines Menschen in den kulturellen Kontexten erweitern, die er durchlebt. Das beginnt mit der Primärsozialisation, bei den im Elternhaus erworbenen Sprachen, und geht über die Sprachen der jeweiligen Gesellschaft bis hin zu Sprachen, die z. B. im Rahmen von Auslandsaufenthalten erworben und/ oder gelernt werden. Vom Lerner/ von der Lernerin aus gedacht, stehen alle diese Sprachen miteinander in Beziehung ${ }^{7}$, sie interagieren miteinander, und gemeinsam bilden sie eine kommunikative Kompetenz, d.h. alle Sprachkenntnisse und Spracherfahrungen eines Menschen zusammen tragen letztlich zu ein und derselben

\footnotetext{
${ }^{5}$ Allerdings ist das empirische Fundament, auf das sich diese häufig zu lesende Feststellung stützt, auch schon ziemlich in die Jahre gekommen (besonders Schläpfer et al. 1991). - Für die konzeptuellen Überlegungen in Kap. 3.4 ist die folgende Präzisierung wichtig: negativ sind die Einstellungen vieler DeutschschweizerInnen in erster Linie gegenüber der eigenen Hochdeutschproduktion - und nicht (so sehr) gegenüber dem Hören des Hochdeutschen.

${ }^{6}$ Einige spezifische Dialektlehrmittel gibt es dagegen schon; sie werden u.a. von Feuz (2001a) aufgeführt. Dort fehlt aber "Los emol" (Müller / Wertenschlag 1985) - vom Ansatz her m.E. auch heute noch das bedeutendste Lehrmittel fürs Schweizerdeutsche.

${ }^{7}$ Sichtbar gemacht hat dieses sprachliche Beziehungsgeflecht bei Kindern auf faszinierende Art Hans-Jürgen Krumm in seinen Mehrsprachigkeitsporträts (cf. Krumm/Jenkins 2000).
} 
kommunikativen Kompetenz bei. Ein wichtiger Aspekt in diesem Konzept von Mehrsprachigkeit ist nun der, dass Menschen in verschiedenen Situationen flexibel auf verschiedene Teile dieser Kompetenz zurückgreifen können sollten, um effektiv miteinander zu kommunizieren. Diese Sicht auf Mehrsprachigkeit bedeutet z. B.: jemand kann - abhängig von den GesprächspartnerInnen, von der Gesprächssituation und abhängig natürlich auch von den Kompetenzen in den verschiedenen Sprachen - von einer Sprache zu einer andern wechseln, und sie bedeutet v. a. auch: jemand kann sich in einer Sprache ausdrücken und die Partnerin/ den Partner in einer anderen verstehen. Und genau das scheint mir der Ort in Mehrsprachigkeitskonzepten zu sein, wo die Dialekte ihren natürlichen Platz schon "haben" (nicht erst zugewiesen bekommen müssen): Bestehende individuelle Mehrsprachigkeit schließt Dialektkompetenzen in vielen Fällen mit ein, denn die Dialekte spielen für die Primärsozialisation in vielen Sprachgemeinschaften eine Rolle. ${ }^{8}$ Weshalb sollten die Dialekte dann, wenn es um Mehrsprachigkeit als übergeordnetes Lernziel geht, unberücksichtigt bleiben? Wären nicht gerade Dialekte und ihre kulturspezifischen Bewertungen ein besonders geeignetes "Tertium Comparationis" zwischen den Ausgangssprachen der Lernenden und der Zielsprache? Wären Dialekte nicht ein prototypischer Gegenstand für interkulturelles Lernen in dem Sinne, dass die Auseinandersetzung mit Einstellungen zum Dialekt in der Kultur der Zielsprache auch zur Reflexion über Funktion und Stellenwert von Dialekten in der Kultur der Ausgangssprache führen könnte? Und, einen grossen Schritt weitergehend (cf. Kap. 3.2) und für spezielle Inlandsituationen gedacht: Bietet es sich nicht an, die Formel "sich in einer Sprache ausdrücken können und den Partner in einer andern verstehen", um die Lesart zu erweitern: sich in einer Standardvarietät, sagen wir: deutschländisches Deutsch, ausdrücken können, und den Partner in einer Nonstandardvarietät, sagen wir: ein alemannischer Dialekt, verstehen können?

\subsubsection{Resultate aus Befragungen von Deutschlernenden}

Eine andere, empirische Begründung für die Integration des Dialekts in den Unterricht sind Resultate aus Befragungen von LernerInnen. Aus der Untersuchung von Baßler/Spiekermann (2001: 19) in Freiburg i. Br. geht hervor, dass sich die Lernenden eine befriedigende bis gute Dialektkompetenz wünschen und dass sie sich hierin viel höhere Ziele stecken als ihre LehrerInnen, die auf die entsprechende Frage antworteten, fast keine oder eine befriedigende Dialektkompetenz würde genügen. ${ }^{9}$ Diese Divergenz ist umso überraschender, als die LernerInnen den Dialekt auf der affektiven Bewertungsdimension tiefer einstufen als die LehrerInnen, sie dürfte sich aber teilweise daraus erklären, dass $80 \%$ der LernerInnen die Fähigkeit, sich deutschen Gesprächspartnern anpassen zu können, für wichtig oder sehr wichtig halten, während nur 20\% der LehrerInnen dieser Meinung sind (ibd.: 19). Immerhin zeigen beide Gruppen ähnliche Schwerpunktsetzungen: Für LernerInnen und LehrerInnen ist das Hörverstehen die für die Dialektkompetenz klar wichtigste Teilfertigkeit (cf. dazu weiter unten unter 3.4).

\footnotetext{
${ }^{8}$ Von den 151 von Baßler/Spiekermann (2001: 13) befragten Lernenden, die aus 48 verschiedenen Ländern kommen, gaben $89 \%$ an, dass in ihrem Herkunftsland Dialekte existieren.

9 Baßler/Spiekermann (ibd.) sprechen von sehr bis hochsignifikanten Unterschieden zwischen den Einschätzungen der LehrerInnen und der LernerInnen, was allerdings auch eine Folge des gewählten statistischen Prüfverfahrens sein dürfte: Beim eingesetzten t-Test handelt es sich sozusagen um das "härteste" aller möglichen Testverfahren für doch eher "weiche", hier mit einem Semantischen Differential erhobene (und ensprechend eher ordinalskalierte) Daten. - Entscheidend ist hier indessen die Tendenz, und die scheint eindeutig zu sein.
} 
Für die Deutschschweiz finden sich einige wenige qualitative Angaben v. a. bei Winiger (2001), die sich auf von der Autorin geleitete, an der Universität Bern durchgeführte Berndeutschkurse beziehen. Winiger hat mittels Fragebögen v. a. die Motivation und den Lernerfolg der Studierenden, die einen Dialektkurs besuchen, erkundet. Hinsichtlich der Motivation kommt sie zum Schluss, dass diese eher integrativen denn instrumentellen Charakter hat. - Dies könnte man durchaus in Zusammenhang mit dem Befund von Baßler/Spiekermann (2001: 19) bringen, wonach sich die meisten LernerInnen ihren Gesprächspartnern anpassen können möchten.

Mit diesen Konstellationen nicht vergleichbar sind dagegen die meisten Auslandsituationen, wie z. B. die Fragebogenstudie von Takahashi (2000) zeigt, die in Tokio bei ca. 300 Studierenden durchgeführt wurde, die Deutsch (als erste oder zweite) Fremdsprache lernen. Hier machen Fragen nach Dialekten des Deutschen gar keinen Sinn, denn zwar wissen immerhin 9 von 10 Studierenden, dass auch in Österreich und in der Schweiz Deutsch gesprochen wird (ibd.: 20), aber es gibt auch Lernende, die glauben, dass ÖsterreicherInnen und SchweizerInnen nur Dialekt sprechen (ibd.: 29). Hier steht wohl primär einmal Information und dann eine gewisse Sensibilisierung für die nationalen Standardvarietäten des Deutschen im Vordergrund, was "zum Verständnis der Varianten der Lehrkörper" und "zur Verstärkung der Lernmotivation beitragen [könnte]" (ibd.).

Ganz offensichtlich ist das Bild, das sich aus diesen nur z. T. miteinander vergleichbaren Untersuchungen ergibt, noch sehr lückenhaft, aber als Tendenz lässt sich für die Inlandsituation doch festhalten, dass es ein - bisher von den DaF-Lehrenden wohl eher unterschätztes - Bedürfnis der Lernenden für die Beschäftigung mit Dialekten gibt, und zwar nicht nur in der Deutschschweiz, und dass dabei integrative Motive eine beachtliche Rolle spielen könnten.

\subsection{Unter welchen Rahmenbedingungen?}

Dass Dialekte im DaF-Unterricht heute wieder ein Thema sind, wurde durch die Diskussionen um das Deutsche als "plurizentrische" oder "pluriareale" Sprache wesentlich begünstigt, wenn nicht sogar ausgelöst. ${ }^{10}$ Diese Diskussionen haben zwar zur Aufwertung der österreichischen und der schweizerischen Standardvarietät beigetragen (für das Schweizerhochdeutsche etwa Bickel 2001) und sie finden ihren Ausdruck nicht nur in plurizentrisch konzipierten Lehrwerken (Beispiel: Moment mal!, Müller et al. 2001), sondern auch in neuen oder revidierten standardisierten, international bekannten Sprachprüfungen (Beispiel: Zertifikat Deutsch).

Dennoch spielt in vielen Lehrmitteln deutschländisches Deutsch nach wie vor die Hauptrolle, besonders auch in den Hörtexten, und da oft in Form eines "neutralen" Standards bzw. einer "unauthentischen Standardleseaussprache". ${ }^{11}$ Daran ist, insoweit es um die Vermittlung und das Lernen produktiver Sprachkompetenzen geht, nichts auszusetzen. Problematisch wird es

\footnotetext{
${ }^{10}$ Wichtige Grundlagenarbeiten aus linguistischer Sicht sind Clyne (1992) und Ammon (1995); sehr aktuell sind Ehlich (2001) (für Deutschland) und Wiesinger (2001) (für Österreich); DaF-bezogene Fragestellungen behandeln z. B. Hensel (2000), Muhr (2000), Ammon (1997), Krumm (1997).

${ }^{11}$ So Baßler/Spiekermann (2002: 31 u. 32) im Rahmen ihrer Analyse zur Berücksichtigung von regionalen Varietäten des Deutschen in DaF-Lehrwerken. Mit "neutralem Standard" und "Standardleseaussprache" ist wohl nördlich geprägtes deutschländisches Deutsch gemeint. Die Analysen der Autoren zeigen allerdings auch eine gewisse Berücksichtigung von Regionalstandards (besonders bundesdeutsche Standardsprache mit bairischer Färbung).
} 
aber, wenn im engern Sinn monozentrische Lehrbuchkonzeptionen und Unterrichtsrealitäten auch den Rahmen für die Entwicklung und das Training rezeptiver Kompetenzen bilden. ${ }^{12}$ Warum? Weil sich unter solchen Bedingungen ein asymmetrisches Verhältnis zwischen dem Sprechen und dem Verstehen einstellt, was die kommunikative Reichweite der Lernervarietäten angeht: Beim Sprechen ist der Kommunikationsradius gross (Lernende können sich im gesamten deutschen Sprachraum verständlich machen, wenn sie eine nationale Standardvarietät beherrschen), beim Verstehen hingegen ist er deutlich kleiner (die Fixierung auf nur eine Standardvarietät wird zu Irritationen führen, wenn Lernende mit einer anderen als der gelernten Varietät konfrontiert sind; cf. dazu auch Takahashi 2000: 22ff.).

Auf einer andern, und zwar auf einer sekundären Ebene steht, dass monozentrische Konzepte im engeren Sinne keine geeignete Voraussetzung für die Behandlung von Dialekten sind: Der Sprung von deutschländischem Deutsch nördlicher Ausprägung zu den Dialekten wäre für die Lernenden viel zu groß, so groß, dass die Dialekte gleichsam als erratische Blöcke, als "Fremdsprachen" gar, empfunden werden müssten (cf. dazu auch Feuz 2001a).

Auf eine weit kohärentere und transparentere Weise lassen sich Dialekte in den Unterricht einbeziehen, wenn die Lernenden zuerst ein Bewusstsein dafür entwickeln können, dass auch die Standardsprache verschieden ausgesprochen wird. Das bedeutet v. a.: die Lernenden sollten (zuerst) Gelegenheit haben, sich in die Variation einer und (dann) in Besonderheiten aller drei nationalen Standardvarietäten einzuhören. Dazu braucht es im Grunde nicht viel. Was es in erster Linie braucht, ist ein breites Angebot an authentischen Hörtexten mit Varianten von deutschländischem, österreichischem und schweizerischem Deutsch, wie man es sich heute ja auch in der Auslandsituation mithilfe der elektronischen Medien oder via Internet recht einfach und weitgehend ortsunabhängig zusammenstellen kann. Mit solchen Hörtexten können die Lernenden erste Erfahrungen mit der Vielfalt des Deutschen machen schon, und darauf kommt es an, auf der Ebene der Standardsprache. Der Aufbau einer Wahrnehmungstoleranz gegenüber Varietäten des Deutschen sollte in jedem Fall bei der Standardsprache beginnen. Das ist ein wichtiges Lehr- und Lernziel für sich, keinesfalls nur eine Vorbereitung für das Dialektverstehen: Ein toleranter Umgang mit den Standardvarietäten im Bereich der rezeptiven Kompetenzen ist primär die Voraussetzung dafür, dass sich die Lernenden in verschiedenen Situationen (beim interaktiven Sprechen ebenso wie beim Hör- und Hörsehverstehen) so verhalten können, dass sie nicht irritiert sind. Darüber hinaus schafft der regelmässige Kontakt mit verschieden ausgeprägten Standardvarietäten dann auch günstige Voraussetzungen für die Behandlung von Dialekten im Unterricht. Als Folge dieses Kontakts sollte sich eine Verstehensdisposition einstellen, die in Bezug auf Variation im sprachlichen Input tolerant ist, und das ist für den Umgang mit Dialekten allgemein und für das Verstehen von Dialekten besonders zentral. Nicht zu unterschätzen sind ferner auch motivationale Aspekte: Wenn sich Lernende beispielsweise mit gesprochenem Schweizerhochdeutsch beschäftigen, kommt früher oder später aus ihrem Kreis die Frage, warum sich denn schweizerische Standardsprache anders anhört als deutschländisches Deutsch, und schon ist man bei den Dialekten. ${ }^{13}$

\footnotetext{
${ }^{12}$ Die Gegenüberstellung von "monozentrischen" und "plurizentrischen" Konzeptionen trägt der üblichen Lehrbuch- und Unterrichtspraxis m.E. zuwenig Rechnung und sollte differenziert werden: "Im engeren Sinn" monozentrisch meint hier, dass nur eine nationale Standardvarietät und diese immer wieder in der gleichen Ausprägung berücksichtigt wird. Davon zu unterscheiden wären monozentrische Konzeptionen "im weiteren Sinn", bei denen zwar z. B. ebenfalls nur deutschländisches Deutsch vorkommt, dieses aber in verschiedenen arealen Ausprägungen (verschiedene "regionale Standards"). Für "plurizentrische" Konzepte bietet sich eine analoge Unterscheidung an (cf. dazu auch die zwei Ebenen der Plurizentrizität bei Muhr, 2000: 31).

${ }^{13}$ Beispiele für die Arbeit mit Standardvarietäten im Unterricht - im "Eigenrecht", aber auch als Sprungbrett für die Beschäftigung mit Dialekten - werden bei Studer (i.Dr.) diskutiert.
} 


\section{3 Auf welchen Stufen?}

Die Frage, ob Dialekte erst auf und ab einem bestimmten Kompetenzniveau berücksichtigt werden sollten, lässt sich letztlich natürlich nur für bestimmte Zielgruppen und deren Interessen und im Inland abhängig auch von der jeweiligen Sprachsituation entscheiden. Wichtige Anhaltspunkte für grundsätzliche Differenzierungen und Eingrenzungen bietet aber der Referenzrahmen des Europarates (2001). Dialekte werden dort an zwei Stellen erwähnt, zum einen im Zusammenhang mit soziolinguistischen Kompetenzen und zum andern im Kontext von interaktiven Aktivitäten und Strategien.

\subsubsection{Soziolinguistische Kompetenzen}

Im Referenzrahmen (Europarat 2001: Kap. 5.2.2.) wird betont, dass nur Teile der Kenntnisse und Fertigkeiten, die zur Bewältigung der sozialen Dimensionen des Sprachgebrauchs erforderlich sind, skaliert (d.h. auf das für die sprachlichen Teilfertigkeiten entwickelte Niveausystem bezogen) werden konnten. Skaliert werden konnten z. B. die sprachliche Kennzeichnung sozialer Beziehungen (u.a. Anredeformeln) und Höflichkeitskonventionen. Abb.1 zeigt Ausschnitte aus der Skala zur "soziolinguistischen Angemessenheit":

\begin{tabular}{|l|l|}
\hline & Soziolinguistische Angemessenheit \\
\hline C2 & $\begin{array}{l}\text { Kann als kompetenter Mittler zwischen Sprechern der Zielsprache und Sprechern aus } \\
\text { seiner eignen Sprachgemeinschaft wirken und dabei soziokulturelle und } \\
\text { soziolinguistische Unterschiede berücksichtigen. }\end{array}$ \\
\hline C1 & $\begin{array}{l}\text { Kann ein grosses Spektrum an idiomatischen und alltagssprachlichen } \\
\text { Redewendungen wieder erkennen und dabei Wechsel im Register richtig } \\
\text { einschätzen; ... } \\
\text { Kann Filmen folgen, in denen viel saloppe Umgangsprache ... vorkommt. }\end{array}$ \\
\hline B2 & $\begin{array}{l}\text { Kann Beziehungen zu Muttersprachlern aufrechterhalten, ohne sie unfreiwillig zu } \\
\text { belustigen, zu irritieren oder sie zu veranlassen, sich anders zu verhalten als bei } \\
\text { Muttersprachlern. } \\
\ldots\end{array}$ \\
\hline B1 & $\begin{array}{l}\text { Ist sich der wichtigsten Unterschiede zwischen den Sitten und Gebräuchen, den } \\
\text { Einstellungen, Werten und Überzeugungen in der betreffenden Gesellschaft und in } \\
\text { seiner eigenen bewusst und achtet auf entsprechende Signale. }\end{array}$ \\
\hline A2 & $\ldots$ \\
\hline A1 & $\ldots$ \\
\hline
\end{tabular}

Abb. 1: Skala "soziolinguistische Angemessenheit" (Referenzrahmen, Europarat 2001: 121122 [Auslassungen stu.]) 
Abgedruckt sind in Abb. 1 nur diejenigen Ausschnitte der Niveaubeschreibungen, die mir für die "Stufenfrage" von Dialektkompetenzen besonders relevant scheinen. In dieser reduzierten Darstellung fällt sofort auf, dass Dialekte erst etwa auf der Mittelstufe eine Rolle spielen, und das in einer spezifischen, nämlich wahrnehmungszentrierten und metakognitiven Weise: Auf der Stufe B1 geht es darum, dass Lernende über ein Bewusstsein für soziokulturelle Besonderheiten verfügen, und zwar nicht nur bezogen auf die Kultur der Zielsprache, sondern auch, und das ist wichtig, in Bezug auf die eigene Gesellschaft. Im Deutschen gehören zu diesen Besonderheiten zweifellos das Verhältnis von Standard- und Nonstandardvarietäten und die damit verbundenen Einstellungen, wie sie oben (Kap. 2) skizziert wurden. Weiter sollten Lernende auf dieser Stufe auf "Signale" achten können, die mit den soziokulturellen Besonderheiten einhergehen. Ein Beispiel dafür könnte etwa das Umsteigen von Dialekt auf Hochdeutsch sein, wie man es z. B. unter DeutschschweizerInnen beobachten kann, wenn eine Deutsche neu zur Gruppe hinzukommt.

Gemäss Abb. 1. sollten die Lernenden dann ab der Stufe B2 zunehmend in der Lage sein, sich auch in soziolinguistischer Hinsicht adäquat zu verhalten. Das kann freilich, bezogen auf die Deutschschweizer Sprachsituation, ganz Verschiedenes heissen. Beispielsweise könnte die für das Niveau B2 typische Kompetenzbeschreibung "Kann Beziehungen zu Muttersprachlern aufrechterhalten, ohne sie unfreiwillig zu belustigen, zu irritieren oder sie zu veranlassen, sich anders zu verhalten als bei Muttersprachlern." so interpretiert werden, dass es in der Tat darum geht, den Lernenden auch eine produktive Dialektkompetenz zu vermitteln. ${ }^{14}$ Ich bin allerdings fest davon überzeugt, dass das eine Interpretation wäre, die weit über das Ziel hinausschiesst. Begründen lässt sich das v. a. auch mit der inhärenten Progression der Niveaubeschreibungen, hier mit den Kompetenzbeschreibungen für die Stufen C1 und C2.

Auf der Stufe C1 stehen ausnahmslos wieder Komponenten des Sprachbewusstseins und rezeptive Fertigkeiten im Vordergrund. Es geht darum, dass Lernende Registerwechsel erkennen und einschätzen können und es geht darum, dass sie Filme verstehen können, in denen Umgangssprache vorkommt. Da nun, wie schon betont, die Umgangssprache in der Deutschschweiz der Dialekt ist, könnte eine Konkretisierung dieser Kompetenzbeschreibung für Deutschlernende in der Schweiz schon so aussehen, dass sie Filme verstehen können sollten, in denen (auch) Dialekt gesprochen wird (wobei dann die Art des Verstehens noch zu präzisieren wäre). Der springende Punkt ist hier aber der: Wenn es richtig ist, dass LernerInnen in der Schweiz auf der Stufe C1 Filme mit Dialekt verstehen können sollten, dann ist es zumindest sehr unwahrscheinlich, dass sie schon auf dem Niveau B2 Dialekt sprechen können sollten.

Stützen lässt sich diese Sichtweise schließlich auch mit der Kompetenzbeschreibung für das Niveau C2, für das Aktivitäten der Sprachmittlung typisch sind und für die wiederum rezeptive Kompetenzen in der Fremdsprache im Zentrum stehen (z. B. souveränes Verstehen eines im Dialekt geführten Gesprächs und zusammenfassende Wiedergabe in schriftlicher oder mündlicher Form in der Ausgangssprache).

\footnotetext{
14 Die Überlegung wäre dann: Unter sich sprechen DeutschschweizerInnen immer Dialekt, also sind Dialektsprechende irritiert, wenn man nicht Dialekt (sondern Hochdeutsch) mit ihnen spricht, also geht es darum, die Fremdsprachigen dazu zu befähigen, dass sie auch Dialekt sprechen können.
} 


\subsubsection{Interaktive Aktivitäten und Strategien}

Dass weiter entwickelte Dialektkompetenzen erst für die höheren und höchsten Kompetenzstufen typisch sind, lässt sich durch die Skala "Muttersprachliche Gesprächspartner verstehen", die zu den Beispielskalen des Referenzrahmens für interaktive Aktivitäten und Strategien gehört, bestätigen (cf. Referenzrahmen des Europarates 2001: Kap. 4.4.3.1). Explizit erwähnt werden Dialekte dort nur auf der Stufe C2, wie Abb. 2 zeigt:

\begin{tabular}{|l|l|}
\hline & Muttersprachliche Gesprächspartner verstehen \\
\hline C2 & $\begin{array}{l}\text { Kann alle muttersprachlichen Gesprächspartner verstehen, auch wenn diese über } \\
\text { abstrakte und komplexe Fachthemen sprechen, die nicht zum eigenen Spezialgebiet } \\
\text { gehören, sofern er/ sie Gelegenheit hat, sich auf einen ungewohnten Akzent oder } \\
\text { Dialekt einzustellen. }\end{array}$ \\
\hline C1 & $\begin{array}{l}\text { Kann im Detail verstehen, wenn über abstrakte, komplexe Themen auf fremden } \\
\text { Fachgebieten gesprochen wird, muss jedoch manchmal Einzelheiten bestätigen } \\
\text { lassen, besonders wenn mit wenig vertrautem Akzent gesprochen wird. }\end{array}$ \\
\hline B2 & $\begin{array}{l}\text { Kann im Detail verstehen, was zu ihm/ ihr in der Standardsprache gesagt wird - } \\
\text { auch wenn es in der Umgebung störende Geräusche gibt. }\end{array}$ \\
\hline B1 & $\ldots$ \\
\hline A2 & $\ldots$ \\
\hline A1 & $\ldots$ \\
\hline
\end{tabular}

Abb. 2: Skala "Muttersprachliche Gesprächspartner verstehen" (Referenzrahmen des Europarates 2001: 80, [Auslassungen und Hervorhebungen stu.])

Die Kompetenzbeschreibung für das Niveau C2 ist sehr komplex. Auf dieser Stufe sollten die Lernenden alle muttersprachlichen GesprächspartnerInnen verstehen können, selbst dann, wenn schwierige Fachthemen besprochen werden, bei denen nicht an einschlägiges Vorwissen angeknüpft werden kann, und wenn Dialekt oder mit ungewohntem Akzent gesprochen wird. Als "mildernder Umstand" für dieses Können wird nur die Gelegenheit zugestanden, sich auf einen ungewohnten Akzent oder Dialekt einzustellen. Und genau das scheint eines der kriterialen Merkmale für den Sprung von C1 auf C2 zu sein (cf. Abb. 2): Typisch für das Niveau C1 sind nämlich gelegentliche Nachfragen zur Verständnissicherung besonders dann, wenn mit wenig vertrautem Akzent über komplexe Themen auf fremden Fachgebieten gesprochen wird.

Nun kann man allerdings sagen, dass die C2-Kompetenz, ein Gespräch unter Muttersprachlern selbst dann verstehen zu können, wenn im Dialekt über unvertraute Fachthemen gesprochen wird, natürlich nicht vom Himmel fällt, sondern auf den vorangehenden Stufen aufgebaut werden sollte - wenn das in einer bestimmten Lernsituation Sinn macht und einem Lernbedürfnis entspricht, was z. B. in der Deutschschweiz der Fall wäre. Die Zerlegung dieser C2-Kompetenz in Teillernziele und deren Zuordnung zu den Stufen B2 und C1 könnte etwa so aussehen: B2 - globales Verstehen von Dialektgesprächen, in denen über alltägliche Themen gesprochen wird; C1 - globales Verstehen von Dialektgesprächen, in denen über komplexe Themen gesprochen wird, oder: detailliertes 
Verstehen von Dialektgesprächen, in denen über alltägliche Themen gesprochen wird. Je nach Lernsituation und Lernbedürfnissen wären genauere Differenzierungen (z. B. für B2 und B2+) möglich und sinnvoll. ${ }^{15}$

\subsection{Welche sprachlichen Teilfertigkeiten?}

$\mathrm{Zu}$ den kontroversen Fragen im Rahmen von Überlegungen zum Einbezug von Dialekten in den DaF-Unterricht gehört zweifellos auch die nach den zu vermittelnden Teilfertigkeiten. Diese Frage wurde bereits in den vorangehenden Abschnitten andiskutiert. Dabei wurde die Position vertreten, dass rezeptive Teilfertigkeiten (mit einem klaren Schwerpunkt auf dem Hörverstehen), vorbereitet und begleitet von metakognitiven Aktivitäten (Entwicklung und Schärfung eines Bewusstseins für soziokulturelle und soziolinguistische Besonderheiten), im Zentrum stehen sollten. Bewusst ausgeklammert bleibt bei dieser Position die Vermittlung von produktiven Dialektkompetenzen. Dieser Aspekt soll im Folgenden noch deutlicher herausgearbeitet und argumentativ besser abgestützt werden, zum einen durch empirische Befunde aus der Untersuchung von Baßler/Spiekermann (2001), zum anderen mit einem sprachpolitischen Argument aus dem und für den Deutschschweizer Kontext.

\subsubsection{Empirische Befunde}

Baßler/Spiekermann (2001: 19) haben ihre ProbandInnen (151 LernerInnen, 15 Lehrende) auch nach den Teilfertigkeiten gefragt, in denen sie sich Dialektkompetenzen wünschen. Vorgegeben waren die Kategorien "sprechen", "schreiben", "hörend verstehen" und "lesend verstehen". Die Resultate sind sehr aufschlussreich: Die einzige Teilfertigkeit, in der sich beide Gruppen eine befriedigende bis gute Dialektkompetenz wünschen, ist das Hörverstehen. Der andere Pol ist das Schreiben, das, ebenfalls für beide Gruppen, mehr oder weniger bedeutungslos ist (alle Werte unter "befriedigend"). Insoweit würden sich also die empirischen Befunde mit der hier vertretenen Position decken.

Anders verhält es sich mit dem Sprechen und mit dem Leseverstehen. In diesen Bereichen wünschen sich die LernerInnen eine befriedigende Kompetenz, wohingegen für die LehrerInnen beide Teilfertigkeiten ähnlich bedeutungslos sind wie das Schreiben. In Bezug auf diese Befunde entspricht meine Position einmal dem Urteil der LernerInnen (befriedigende Kompetenz beim Leseverstehen), das andere Mal dem Urteil der LehrerInnen (produktive Dialektkompetenz im mündlichen Bereich unbedeutend). Dies verlangt nach zusätzlichen Begründungen:

Was das Leseverstehen betrifft, so scheint mir der Wunsch der LernerInnen, auch in diesem Bereich eine befriedigende Dialektkompetenz zu erreichen, verständlich und sinnvoll vorausgesetzt, schriftliche Dialekttexte spielen eine gewisse Rolle, was z. B. für die Deutschschweiz zutrifft.

Demgegenüber kann ich - aus der Sicht eines Lehrers - kein Argument erkennen, das für die Vermittlung von produktiven Dialektkompetenzen spräche - es sei denn, man wolle die ganze Verantwortung für die sprachliche Anpassung in der Kommunikation zwischen

\footnotetext{
${ }^{15}$ Bei einem in dieser Art "flexiblen" Umgang mit den Skalen des Referenzrahmens ist allerdings zu bedenken, dass die Zerlegung von komplexen Kompetenzen in Teilkompetenzen und deren Zuordnung zu vorangehenden Niveaus primär für den spezifischen Lernkontext gültig sind, für den sie vorgenommen wurden. Verallgemeinerungen mit dem Ziel, die resultierenden Teilkompetenzen für ein bestimmtes Niveau als typisch auszuweisen, würden grössere empirische Untersuchungen voraussetzen.
} 
DialektsprecherInnen und Fremdsprachigen einseitig den Fremdsprachigen überbürden. ${ }^{16}$ Geht man dagegen davon aus, dass Anpassung (wenigstens im sprachlichen Bereich) ein zweiseitiger Prozess sein sollte, ist das ein erstes und zentrales Argument gegen die Vermittlung von produktiven Dialektkompetenzen: DialektsprecherInnen können die Standardsprache ja in aller Regel problemlos verstehen, weshalb sollten sie denn ihre rezeptive Hochdeutschkompetenz nicht in die Kommunikation mit Fremdsprachigen einbringen $?^{17}$ Ausserdem werden durch das Sprechenlernen des Dialekts m.E. Ressourcen gebunden, die mit weit mehr Gewinn zum Ausbau produktiver Fertigkeiten in der Standardsprache oder auch für das Erlernen einer weiteren Fremdsprache genutzt werden könnten. Und schliesslich stellt sich - mit Blick auch auf die Binnenmigration in den deutschsprachigen Ländern - die Frage, welchen Dialekt die Fremdsprachigen denn sprechen lernen sollten (cf. dazu unten, 3.5).

\subsubsection{Sprachpolitische Argumentation}

Gegen die Vermittlung produktiver Dialektkompetenzen in Deutschschweizer Lernsituationen - und für einen selbstbewussteren Umgang der DeutschschweizerInnen mit dem gesprochenen Hochdeutsch - lässt sich auch ein sprachpolitisches Argument anführen, das Haas 2001 im Rahmen einer sprachgeschichtlichen Betrachtung zur Entstehung der deutschen Standardsprache entwickelt. Haas (ibd.: 17f.) kann u.a. zeigen, dass das Hochdeutsche nicht einfach eine in die Schweiz importierte Sprache ist, sondern dass Schweizer durchaus einen gewissen Anteil an der Entwicklung der deutschen Gemeinsprache hatten. Er fragt dann: "Was gehen verfehlte Vorstellungen über die Entstehung der deutschen Gemeinsprache die gewöhnlichen Sprecher an? Nichts - so lange sie akademisch bleiben. Viel - wenn sie in die Sprachideologie einsickern und das Verhalten beeinflussen. Nehmen wir die Idee von der Fremdheit und Importiertheit der deutschen Gemeinsprache. Die Idee ist falsch, und das könnte den Laien kühl lassen; aber sie übertreibt auch die sprachliche und psychologische Kluft zu unserer Gemeinsprache, auf die wir bedingungslos angewiesen sind. Und das kann niemanden kühl lassen." Haas (ibd.: 18) schliesst seine Betrachtungen mit folgender Forderung: "Sprachpolitik in diesem Landesteil [gemeint ist die Deutschschweiz, stu.] muss die psychologischen und institutionellen Hürden abbauen, die uns daran hindern, unsere Gemeinsprache selbstbewusst zu sprechen - bei jenen Gelegenheiten, in denen die Funktionalität einleuchtet." Dieser Forderung schliesse ich mich vollumfänglich an. Und wäre nicht gerade der Kontakt mit Fremdsprachigen ein prototypisches Beispiel für eine Gelegenheit, in der die Funktionalität, Hochdeutsch zu sprechen, eigentlich jeder Deutschschweizerin und jedem Deutschschweizer einleuchten müsste?

\footnotetext{
${ }^{16}$ Aus der Sicht von Lernenden mag es, wie auch die Befragung von Winiger 2001 nahelegt, in der Tat manchmal das Bedürfnis geben, sich auch produktive Dialektkompetenzen anzueignen.

${ }^{17}$ Feuz 2001a verweist in diesem Zusammenhang auf die negativen Einstellungen der DeutschschweizerInnen gegenüber dem gesprochenen Hochdeutsch: DeutschschweizerInnen würden gewissen Gesprächssituationen ausweichen, wenn nicht so gesprochen werden könne, wie ihnen der Schnabel gewachsen sei. (ibd.: 3). M.E. ist das aber gar kein Argument für produktive, sondern für rezeptive Dialektkompetenzen: Um an einem Gespräch unter DeutschschweizerInnen teilzunehmen, brauchen Fremdsprachige jedenfalls auch dann nicht Dialekt sprechen zu können, wenn die DialektsprecherInnen "so sprechen, wie ihnen der Schnabel gewachsen ist". Nein, was Fremdsprachige in solchen Situationen brauchen, ist eine relativ weit entwickelte Kompetenz im Hörverstehen von Dialekten (cf. auch Fussnote 5).
} 


\subsection{Welche Dialekte?}

Wer produktive Dialektkompetenzen unterrichten möchte, kommt nicht umhin, sich für einen Dialekt zu entscheiden. ${ }^{18}$ Dabei stellen sich eine ganze Reihe von Fragen, die sich m.E. nicht befriedigend beantworten lassen, auch dann nicht, wenn man sich auf das Prinzip des Lernortes beruft, denn die Gleichung "ein Lernort - ein Dialekt" dürfte heute nur noch in Ausnahmefällen aufgehen. Ist die Situation heute im Zeichen von Mobilität und Migration weltweit, aber eben auch innerhalb des deutschsprachigen Raumes - nicht vielmehr die, dass an einem Ort mehrere Dialekte zusammenkommen? Und wenn dem so ist, was mindestens auf die grösseren Deutschschweizer Städte (und da besonders auch auf das "studentische Umfeld") zutreffen dürfte, was wären dann die Kriterien für die Auswahl eines bestimmten Dialekts? Die relative Grösse des generell kleinen Kommunikationsradius? Die "Mehrheitsverhältnisse" vor Ort? Der linguistische Abstand zur Standardsprache? Die Konnotationen (positive oder neutrale Einstellungen, Heterostereotyp, Autostereotyp...)? Oder, und auf einer ganz anderen Ebene: die Art und der Umfang des "Anschauungsmaterials", das es für einen Dialekt gibt? Oder wäre schlicht und einfach der Dialekt der Lehrperson ausschlaggebend?

Nein, ein bedeutendes Merkmal des heutigen Deutsch ist doch die Vielfalt gesprochener Varietäten (z. B. Ehlich 2001), und dazu gehört - als Teilmenge gleichsam - die Vielfalt klein- und, in der Deutschschweiz, kleinsträumiger Dialekte. Und eben dieser Vielfalt kann man m.E. dann wenigstens in Ansätzen Rechnung tragen, wenn man sich auf die Vermittlung rezeptiver Dialektkompetenzen beschränkt. Dann ist es möglich, mehrere Dialekte zu thematisieren, und das wiederum erlaubt es, auf so etwas wie eine allgemeinere rezeptive Kompetenz im Bereich der Nonstandardvarietäten hinzuarbeiten. Eine solche allgemeinere rezeptive Varietätenkompetenz umfasst einerseits ein Verständnis für kulturelle und sprachliche Besonderheiten von verschiedenen Dialekten und andererseits die Fertigkeit, ausgewählte Dialekte (vornehmlich global) verstehen zu können. Dialekte auswählen muss man also auch bei einem auf die Rezeption beschränkten Konzept, und auch die AuswahlKriterien dürften in etwa die gleichen sein. Nur, und das ist ein wesentlicher Unterschied: bei dem hier vorgeschlagenen Konzept muss die Entscheidung für bestimmte Dialekte weder stillschweigend vorausgesetzt noch allein durch die Lehrperson getroffen werden, sondern sie kann in Absprache mit den LernerInnen erfolgen, und dabei können verschiedene der angeführten Kriterien auf verschiedene Dialekte angewendet werden (was bereits viel interessanten Gesprächsstoff hergibt).

Die Vorteile des Auf- und Ausbaus einer weiter gefassten, allgemeineren rezeptiven Dialektkompetenz liegen m.E. auf der Hand: Es entsteht kein Bruch mit dem in verschiedenen Kontexten immer wieder betonten Bedürfnis der Lernenden, das Gelernte möglichst weiträumig einsetzen zu können, es lässt sich teilnehmerzentriert unterrichten auch in dem Sinne, dass Dialekte aufgegriffen werden können, mit denen die LernerInnen bereits Erfahrungen gemacht haben oder die sie aus andern Gründen interessieren, es spielt keine Rolle, welchen Dialekt die Lehrperson spricht (und mit welchen arealen, sozialen... Varianten sie diesen spricht) und schliesslich, und das ist vielleicht der wichtigste Aspekt: eine allgemeinere rezeptive Varietätenkompetenz ist ein "Startkapital" für neue Lernumgebungen

\footnotetext{
${ }^{18}$ Bei Feuz 2001a, b und bei Winiger 2001 fällt die Wahl auf Berndeutsch. Ich wollte das für meine Zielgruppen (Studierende an der Universität Freiburg/Fribourg) nicht entscheiden: Die eine Lernerin arbeitet an der Kasse im Supermarkt, ein anderer an der Tankstelle - beide kommen mit verschiedenen Dialekten in Kontakt. Eine dritte Lernerin hat einen Freund in Zürich, die vierte hat zuvor in München studiert, zwei Studentinnen aus den Niederlanden haben sich einer Gruppe Studierender aus Luzern angeschlossen...
} 
mit noch unbekannten Dialekten und (dadurch auch) eine Art "Mitgift" für das selbständige Weiterlernen.

\subsection{Wie?}

Das methodische Prinzip, das sich zur Umsetzung des vorgeschlagenen Konzepts im Unterricht m.E. am besten eignet, lautet: "Dialekte verstehen - Hochdeutsch sprechen und schreiben". Entwickelt und zuerst praktiziert wurde dieses Prinzip von Müller/Wertenschlag (1985). ${ }^{19}$

Ein auf die Deutschschweizer Lernsituation bezogenes Beispiel soll zeigen, wie dieses Prinzip funktioniert, wobei es mir hier nur auf die Illustration der Verbindung von Aktivitäten im Unterricht einerseits und den dabei geübten Teilfertigkeiten und fokussierten Varietäten andererseits ankommt (nicht auf Einzelheiten einer konkrete "Dialektdidaktik"). Das Beispiel ist eng an Ideen aus Los emol, dem leider vergriffenen Lehrbuch von Müller/Wertenschlag (1985), angelehnt:

\begin{tabular}{|c|c|c|}
\hline & Aktivitäten im Unterricht & $\begin{array}{l}\text { Fokussierte } \\
\text { Teilfertigkeiten und } \\
\text { Varietäten }\end{array}$ \\
\hline a) & $\begin{array}{l}\text { Austausch über (sprachliche) Erfahrungen im Kontakt } \\
\text { mit DeutschschweizerInnen (zuerst in Gruppen, dann } \\
\text { im Plenum) }\end{array}$ & Standardsprache sprechen \\
\hline \multirow[t]{2}{*}{ b) } & $\begin{array}{l}\text { Die LernerInnen hören einen Vortrag zum Thema } \\
\text { "Dialekt und Hochdeutsch in der Deutschschweiz". }\end{array}$ & Dialekt hören \\
\hline & $\begin{array}{l}\text { Während des Hörens haben sie das standardsprachliche } \\
\text { Transkript des Hörtextes vor sich und können mitlesen. }\end{array}$ & Standardsprache lesen \\
\hline \multirow[t]{2}{*}{ c) } & $\begin{array}{l}\text { Die Lernenden hören den Vortrag ein zweites Mal, jetzt } \\
\text { aber ohne Transkript. }\end{array}$ & Dialekt hören \\
\hline & $\begin{array}{l}\text { Während des Hörens haben sie einen Raster mit } \\
\text { Stichworten zu wesentlichen Inhaltspunkten des } \\
\text { Vortrags vor sich, den sie ausfüllen sollen. }\end{array}$ & Standardsprache schreiben \\
\hline d) & $\begin{array}{l}\text { Die Lernenden vergleichen die teilweise ausgefüllten } \\
\text { Raster und ergänzen sie in Gruppen. }\end{array}$ & $\begin{array}{l}\text { Standardsprache sprechen } \\
\text { und schreiben }\end{array}$ \\
\hline e) & $\begin{array}{l}\text { Plenumsdiskussion über die Deutschschweizer } \\
\text { Sprachsituation anhand des Rasters. }\end{array}$ & Standardsprache sprechen \\
\hline
\end{tabular}

Abb. 3: Beispiel für das Prinzip "Dialekt verstehen - Hochdeutsch sprechen und schreiben"

Wenn man zum ersten Mal mit diesem Prinzip arbeitet, kann es bei den LernerInnen bei Schritt b) zu leichten Irritationen kommen. ${ }^{20}$ Diese Verbindung ist als Brücke gedacht, die das

\footnotetext{
${ }^{19}$ Ich war sachlich positiv überrascht, von diesem Prinzip, das ich auf der IDT 2001 in Luzern vorgestellt hatte, jetzt auch in Baßler/Spiekermann (2001) zu lesen. Da bei letzteren aber der einschlägige Literaturhinweis fehlt, stelle ich das Prinzip hier noch einmal vor.

${ }^{20}$ Wobei es sich bei dieser Art der Verbindung von rezeptiven Aktivitäten - Dialekt hören und dabei in der Standardsprache mitlesen -um eine Rezeptionshaltung handelt, die durchaus eine Entsprechung in der kommunikativen Realität ausserhalb des Klassenzimmers hat: Werden Deutschschweizer Dialektsendungen im deutschen Fernsehen gezeigt, sind Untertitel in der Standardsprache zunehmend die Regel.
} 
nicht oder noch wenig Bekannte (Dialekt) im Vertrauten (Standardsprache) verankert. Erfahrungsgemäss verschwinden die anfänglichen Irritationen schnell, d.h. die LernerInnen empfinden diese Brücke als sehr hilfreich und sie nützen sie bald auch kreativ in dem Sinne, dass sie selber nach Korrespondenzen zwischen Dialekt und Standardsprache zu forschen beginnen. Bei weiter fortgeschrittenen Kenntnissen des behandelten Dialekts kann dieser Zwischenschritt auch modifiziert (reduziert) oder übersprungen werden.

Das Prinzip "Dialekte verstehen - Hochdeutsch sprechen und schreiben" lässt sich sehr flexibel handhaben: Es lässt sich auf beliebige Dialekte anwenden, man kann ein breites Spektrum von Textsorten und Themen berücksichtigen und man kann die Instruktionen und Antwortformate je nach Unterrichtsziel fast beliebig variieren und die einzelnen Aufgaben auch in grössere Zusammenhänge einbetten. Ausweitungen sind insbesondere auch in Richtung projektorientierten Arbeitens möglich (cf. auch Studer, i.Dr.). Wesentlich für dieses Prinzip sind im Grunde nur zwei Konstanten: der Input - gewählt werden sollte wenn immer möglich ein authentischer Dialekttext, wofür primär Hörtexte, aber auch audiovisuelle Texte und, je nach Stellenwert in der jeweiligen Sprachsituation, auch Lesetexte in Frage kommen und die darauf bezogenen Aktivitäten in der Standardsprache.

\subsection{In welchem Umfang?}

Abschliessend soll noch die Frage nach dem Stellenwert, den Dialekte im Rahmen des DaFUnterrichts haben könnten, angesprochen werden. Verbunden damit ist auch die Frage nach den "Gefässen", die sich für die Beschäftigung mit Dialekten eignen. Die Kriterien für die Entscheidung dieser Fragen liegen auf der Hand: Ausschlaggebend sind der Lernort und die Bedürfnisse und Ziele der Lernenden.

In der Deutschschweiz, für die die hohe Relevanz der Dialekte in der mündlichen Kommunikation charakteristisch ist (cf. oben, Kap. 2), ist zweifellos ein Angebot an speziellen Varietätenkursen, in denen die Dialekte eine Hauptrolle spielen, angezeigt. Das allein reicht aber m.E. nicht aus: Will man am übergeordneten Ziel der Vermittlung einer gebrauchsfähigen Sprache festhalten, sollten Aspekte der Dialektthematik vermehrt auch in das übliche Programm von allgemeinen Sprachkursen integriert werden (zur "Stufenfrage" cf. oben, Kap. 3.3). Das hier entwickelte Konzept zum Aufbau einer allgemeineren rezeptiven Varietätenkompetenz versteht sich als Orientierungshilfe für beide Arten von Kursen, denn zwar dürfte das Verstehenlernen von Dialekten in speziellen Varietätenkursen eine Hauptrolle spielen, aber bezüglich der Inhalte (cf. Kap. 3.1 u. 3.5) und Arbeitsformen (cf. Kap. 3.6) sehe ich doch viele Gemeinsamkeiten, und der Unterschied ist m.E. primär ein solcher der Dauer und Intensität der Auseinandersetzung mit Dialekten. Was die Frage der Stundendotation betrifft, scheinen mir die Hinweise bei Feuz 2001a (5) und Winiger 2001 (40) wichtig, wonach man in einsemestrigen Spezialkursen mit einer Doppelstunde pro Woche sehr viel erreichen kann. Diese Erfahrungen kann ich nur bestätigen. Darüber hinaus aber zeigen meine Erfahrungen, dass man auf der Basis des vorgestellten Konzepts auch im Rahmen von allgemeinen Sprachkursen einen Grundstein für das Dialektverstehen legen kann, und das schon mit einem kleinen Projekt, das lediglich etwa vier Kursstunden beansprucht. ${ }^{21}$

\footnotetext{
${ }^{21}$ Und dafür braucht es m.E. keineswegs fundierte dialektologische Kenntnisse auf Seiten der Lehrenden, wie sie Baßler/Spiekermann 2002, 34f. offenbar vorschweben: "Voraussetzung für eine Übertragung des oberrheinischalemannischen Lautstandes in den standarddeutschen ist eine gezielte und selektive Vermittlung von phonologischem und grammatischem Wissen." (ibd.: 35) Für wen und wofür genau bräuchte es denn solches Wissen? Sicher nicht für "meine" LernerInnen. Die merken nämlich bereits in der ersten Kursstunde oder schon vorher und jedenfalls von sich aus, dass "Huus" (schweizerdeutsch) "Haus" (standarddeutsch) entspricht. Und
} 
Diese Erfahrungswerte bieten vielleicht auch Anhaltspunkte für Lernsituationen in den andern deutschsprachigen Ländern. In Lernorten Deutschlands und Österreichs, in denen der Dialekt eine weniger dominante Stellung einnimmt und mit heterogeneren Einstellungen verbunden ist als in der Deutschschweiz, dürften rezeptive Kompetenzen im Bereich der regionalen Standards und Regionalsprachen eher wichtiger sein als Dialektkompetenzen. Entsprechend könnten hier allgemeine Sprachkurse, in die Aspekte der Dialektthematik integriert sind, in vielen Fällen ausreichen.

Letzteres gälte dann besonders auch für nichtdeutschsprachige Länder: In der Auslandsituation muss man über spezielle Varietätenkurse gar nicht diskutieren. Ein Angebot an allgemeinen Sprachkursen aber, in denen Dialekte im Rahmen von "landeskundlichen Fenstern" ein Thema sind und in denen dann v. a. die soziokulturellen Dimensionen von Dialekten im Vordergrund stehen könnten, scheint mir auch in der Auslandsituation durchaus sinnvoll. Weit sinnvoller wäre das jedenfalls, als anhand von mittelmäßig didaktisierten Texten Länderstereotype zu wiederholen. Ein Dialektlied etwa als Beispiel für einen authentischen Hörtext gibt viel mehr her als Kühe, Käse und Konsorten - in soziokultureller Hinsicht zunächst und dann auch, bei Zielgruppen, die sich für einen Aufenthalt im deutschsprachigen Raum vorbereiten, in sprachlicher Hinsicht.

\section{Bilanz}

Überblick man nun die Überlegungen in den Abschnitten 3.1 bis 3.7, so lassen sich die Konturen des Konzepts für einen Erwerb des Deutschen als Fremdsprache, der sich im Bereich der rezeptiven Kompetenzen auch auf Dialekte erstreckt, doch klarer erkennen. Die Beschäftigung mit Dialekten im DaF-Unterricht kann zusammenfassend und thesenartig wie folgt begründet und präzisiert werden:

- Empirische Untersuchungen und Beobachtungen in der Inlandsituation weisen darauf hin, dass es ein bisher von den Lehrenden eher unterschätztes Bedürfnis der Lernenden gibt, sich mit Dialekten zu beschäftigen.

- Dialekte gehören in vielen Fällen zu den frühen sprachlichen Erfahrungen von Fremdsprachigen in ihren Herkunftsländern. Dies macht die Dialekte zu einem geeigneten Gegenstand für interkulturelles Lernen: die Auseinandersetzung mit Dialekten und den damit verbundenen Einstellungen in der Zielsprache kann das Nachdenken über Funktion und Stellenwert von Dialekten in der Kultur der Ausgangssprache anstossen.

- Ein bedeutendes Lernziel für sich und gleichzeitig eine wesentliche Voraussetzung für den kohärenten Einbezug von Dialekten in den DaF-Unterricht ist der Aufbau einer Wahrnehmungstoleranz gegenüber (Ausprägungen von) nationalen Varietäten der deutschen Standardsprache.

- Dialektkompetenzen gehören grundsätzlich zu den höheren und höchsten Niveaus, die LernerInnen in der Fremdsprache erreichen. Je nach Stellenwert, den die Dialekte an einem bestimmten Lernort haben, ist es jedoch sinnvoll, Dialekte auf und ab der Mittelstufe zu berücksichtigen.

- Geeignete Gefässe für den Einbezug von Dialekten in den DaF-Unterricht sind, besonders in der Deutschschweiz, spezielle Varietätenkurse, aber auch - und das dann weitgehend

was wäre, wenn die Lernenden, wie meine Beobachtungen (Studer, i.Dr.) vermuten lassen, bei Umsetzungsunsicherheiten eher semantisch vorgehen als morphologisch und dabei sämtliche ko- und kontextuelle Indizien ausnützen? Natürlich kann der eine oder andere strukturelle und sprachgeschichtliche Hinweis in bestimmten Lernphasen hilfreich sein, aber entscheidend ist m.E. ein entdeckendes oder forschendes Lernen - und dabei müssen die Lehrenden durchaus keinen riesigen Wissensvorsprung haben. 
unabhängig vom Lernort - allgemeine Sprachkurse, in die Aspekte der Dialektthematik integriert sind.

- Bei der Vermittlung von Dialektkompetenzen sollten rezeptive Teilfertigkeiten im Zentrum stehen. Am wichtigsten ist das Hörverstehen.

- Wird von der Vermittlung produktiver Dialektkompetenzen abgesehen, werden Lernpotentiale freigesetzt, die mit Gewinn zur Erweiterung produktiver Hochdeutschkompetenzen oder auch für das Erlernen einer weiteren Fremdsprache genutzt werden können.

- Eine Beschränkung auf die Vermittlung rezeptiver Dialektkompetenzen erlaubt es auch, mehrere Dialekte zu thematisieren und so auf eine allgemeinere rezeptive Kompetenz im Bereich der Nonstandardvarietäten hinzuarbeiten. Diese umfasst das Verstehen (mindestens) eines Dialekts und die Fähigkeit, weitere Dialekte verstehen zu lernen.

- Ein einfaches Prinzip, das sich gut zur Vermittlung rezeptiver Dialektkompetenzen eignet, und das vielfältig variiert werden kann, lautet: "Dialekte verstehen - Hochdeutsch sprechen und schreiben".

Die Intention dieses Konzepts ist nicht Revolution, sondern Evolution des DaF-Unterrichts. Es geht darum, einen Orientierungsrahmen bereit zu stellen, der es erlaubt, den Dialekten als Teil der Vielfalt des Deutschen im Unterricht positiv, aber auch reflektiert zu begegnen, und das heisst v. a. auch: kohärent zum üblichen Programm und transparent für die LernerInnen. Der Orientierungsrahmen sagt nur, wie dies erfolgen könnte und bietet dazu eine Reihe von Kriterien an, die zu entscheiden oder auch zu hinterfragen natürlich Sache jeder einzelnen Lehrerin und jedes einzelnen Lehrers ist.

\section{$5 \quad$ Literatur}

Ammon, Ulrich (1997): Die nationalen Varietäten des Deutschen im Unterricht von Deutsch als Fremdsprache. In: Jahrbuch Deutsch als Fremdsprache 23, 1997. Herausgegeben von Alois Wierlacher et. al. München: Iudicium, 141-158.

Ammon, Ulrich (1995): Die deutsche Sprache in Deutschland, Österreich und der Schweiz. Das Problem der nationalen Varietäten. Berlin: de Gruyter.

Baker, Colin (1992): Attitudes and Language. Clevedon etc.: Multilingual Matters..

Baßler, Harald/ Spiekermann, Helmut (2002): "Regionale Varietäten des Deutschen im Unterricht Deutsch als Fremdsprache (II)." Deutsch als Fremdsprache 1/02, 31-35. [Teil I in: Deutsch als Fremdsprache 4/01, 205-213; zusammen zitiert als Baßler/Spiekermann 2002]

Baßler, Harald/ Spiekermann, Helmut (2001): "Dialekt und Standardsprache im DaFUnterricht. Wie Schüler urteilen - wie Lehrer urteilen." Linguistik online 9, 2/01.

Bickel, Hans (2001): "Schweizerhochdeutsch: kein minderwertiges Hochdeutsch! Das Deutsche als plurizentrische Sprache aus Schweizer Sicht." Babylonia 2/01: 19-22.

Clyne, Michael (1992): "German as a Pluricentric Language." In: Clyne, Michael (ed.): Pluricentric Languages. Differing Norms in Different Nations. Berlin, de Gruyter: 117147.

Council of Europe (ed.) (1998): Modern Languages: Learning, Teaching, Assessment. A common European Framework of reference. Strassbourg.

Ehlich, Konrad (2001): "Standard zwischen Bühne und Regionalität." In: Ehlich, Konrad/Ossner, Jakob/Stammerjohann, Harro (eds.): Hochsprachen in Europa. Entstehung, Geltung, Zukunft. Freiburg i.Br., Fillibach: 145-158. 
Europarat (2001): Gemeinsamer europäischer Referenzrahmen für Sprachen: Lernen, lehren und beurteilen. Herausgegeben vom Goethe-Institut, Inter Nationes, der KMK, der EDK und dem BMBWK. Berlin etc.

Feuz, Barbara (2001a): "Dialektale Varietät als Fremdsprache unterrichten. Ein Erfahrungsbericht." Linguistik online 9, 2/01.

Feuz, Barbara (2001b): "Warum nicht mal Schweizerdeutsch im DaF-Unterricht? In: Babylonia 2/01: 42-44.

Flüe-Fleck, Hans Peter von/Hove, Ingrid (1994): "Schweizerhochdeutsch: ein Thema im Unterricht Deutsch als Fremdsprache?" In: Breitung, Horst (ed.): Phonetik, Intonation, Kommunikation. München: 49-68.

Goethe-Institut, Österreichisches Sprachdiplom Deutsch, Schweizerische Konferenz der kantonalen Erziehungsdirektoren, Weiterbildungs-Testsysteme GmbH (eds) (1999): Zertifikat Deutsch. Lernziele und Testformat. Frankfurt a.M.

Grüezi mitenand. Luzernerinnen und Luzerner erzählen von sich und ihrer Stadt. Eine CDROM zum Schweizerdeutschen. Konzept: Monika Clalüna und Bruno Frischherz. Produktion: Brunner AG, Kriens (produziert für die IDT 2001 in Luzern).

Haas, Walter (2001): "Sonderfall oder nicht - das ist die Frage". Babylonia 2/0: 16-18.

Haas, Walter (2000): "Die deutschsprachige Schweiz." In: Bickel, Hans/Schläpfer, Robert, (eds.): Die viersprachige Schweiz. Aarau: Sauerländer.

Hensel, Sonja (2000): "Welches Deutsch sollen wir lehren?" Zielsprache Deutsch 31: 31-39.

Hoberg, Rudolf (2000): "Sprechen wir bald alle Denglisch oder Germeng?" In: EichhoffCyrus, Karin M./Hoberg, Rudolf (eds.): Die deutsche Sprache zur Jahrtausendwende. Sprachkultur oder Sprachverfall? Mannheim etc.: 303-316.

Hove, Ingrid (2002): Die Aussprache der Standardsprache in der deutschen Schweiz. Tübingen: Niemeyer.

Klauer, Karl (1988): Einstellungen. Der Einfluss der affektiven Komponente auf kognitive Urteile. Hamburg: Hogrefe.

Krumm, Hans-Jürgen/ Jenkins, Eva-Maria (eds.) (2001): Kinder und ihre Sprachen lebendige Mehrsprachigkeit. Sprachenporträts. Gesammelt und kommentiert von HansJürgen Krumm. Wien.

Krumm, Hans-Jürgen (1997): Welches Deutsch lehren wir? Einführung in den thematischen Teil. In: Jahrbuch Deutsch als Fremdsprache 23, 1997. Herausgegeben von Alois Wierlacher et. al. München: Iudicium, 133-139.

Maurer, Ernst (2000): Deutsch in der Schweiz. Ein Sprachkurs für Erwachsene und Jugendliche. Zug: Klett.

Müller, Martin et al. (1996-98): Moment mal! Lehrwerk für Deutsch als Fremdsprache. 3 Bde. München: Langenscheidt.

Müller, Martin/ Wertenschlag, Lukas (1985): Los emol. Schweizerdeutsch verstehen. Zürich: Langenscheidt.

Muhr, Rudolf (2000): Österreichisches Sprachdiplom Deutsch. Lernzielkataloge zu Basisformulierungen, Lexik, Sprechhandlungen, Höflichkeitskonventionen, Diskurs und Diskursstrukturen, Deutsch als plurizentrische Sprache. Wien.

Pilet, Jacques (2001): "On ne parle pas l'allemand en Suisse." Babylonia 2/01: 8-10.

Schläpfer, Robert, et al. (1991): Das Spannungsfeld zwischen Mundart und Standardsprache in der deutschen Schweiz: Spracheinstellungen junger Deutsch- und Welschschweizer; eine Auswertung der Pädagogischen Rekrutenprüfung 1985. Aarau: Sauerländer.

Schmidlin, Regula Madeleine (1999): Wie Deutschschweizer Kinder schreiben und erzählen lernen. Textstruktur und Lexik von Kindertexten aus der Deutschschweiz und aus Deutschland. Tübingen: Francke. 
Siebenhaar, Beat/Wyler, Alfred ( $\left.{ }^{5} 1997\right):$ Dialekt und Hochsprache in der deutschsprachigen Schweiz. Zürich: Pro Helvetia

Studer, Thomas (im Druck): "Varietäten des Deutschen verstehen lernen. Überlegungen und Beobachtungen zum universitären DaF-Unterricht." Erscheint in: Häcki Buhofer, Annelies (ed.): Spracherwerb und Lebensalter.

Takahashi, Hideaki (2000): Verschiedene Varietäten des Deutschen und deren Beziehung zum Unterricht Deutsch als Fremdsprache (DaF). Zeitschrift für Angewandte Linguistik 32: 19-33.

Werlen, Iwar (1998): "Mediale Diglossie oder asymmetrische Zweisprachigkeit? Mundart und Hochsprache in der deutschen Schweiz." Babylonia 1/98: 22-35.

Wiesinger, Peter (2001): "Die deutsche Schrift- und Standardsprache in Österreich. Stellung, Beurteilung, Perspektiven." In: Ehlich, Konrad/ Ossner, Jakob/ Stammerjohann, Harro (eds.) Hochsprachen in Europa. Entstehung, Geltung, Zukunft. Freiburg i. Br., Fillibach: 159-169.

Winiger, Elisabeth (2001): "'Wenn ich nur mit Berner bin, dann fühle ich mich verloren'. Schweizerdeutsch unterrichten. Ein Erfahrungsbericht über einen Berndeutschkurs an der Universität Bern." Babylonia 2/01: 38-41. 